\title{
Effect of Acoustic Doppler Velocimeter Sampling Volume Size on Measurements of Turbulence
}

\author{
MoHAmmad KAZEMI, ${ }^{\mathrm{a}}$ BABAK KHORSANDI, ${ }^{\mathrm{a}}$ AND LAURENT MydLARSKI ${ }^{\mathrm{b}}$ \\ ${ }^{a}$ Department of Civil and Environmental Engineering, Amirkabir University of Technology (Tehran Polytechnic), Tehran, Iran \\ ${ }^{\mathrm{b}}$ Department of Mechanical Engineering, McGill University, Montreal, Quebec, Canada
}

(Manuscript received 27 October 2020, in final form 3 December 2020)

\begin{abstract}
The relatively large sampling volume of acoustic Doppler velocimeters (ADVs) is expected to influence their measurement of turbulence. To study this effect, a series of experiments using different sampling volume sizes was conducted in an axisymmetric turbulent jet. The results show that the mean velocities are not significantly affected by the size of the sampling volume. On the other hand, reducing the sampling volume size results in an increase in the variances of the $u$ and $v$ velocities, while its effect on the variance of the $w$ velocity is negligible. Application of a noise-reduction method to the data renders the velocity variances nearly independent of sampling volume size, suggesting that the difference was mainly due to Doppler noise. The principal conclusion of this work is, therefore, that - as long as the characteristic length of sampling volume is much smaller than the integral length scale of flow-increasing the sampling volume size (i.e., increasing spatial averaging over highly correlated scatterers) can reduce Doppler noise and result in more accurate measurements of the velocity variances. Application of noise-reduction methods to the data is found to be especially important when the sampling volume size is reduced to capture smaller scales, or for near-boundary measurements. Furthermore, noise due to mean velocity shear, even at the largest velocity gradient along the jet radial profile, is found to be negligible in the present work.
\end{abstract}

KEYWORDS: Acoustic measurements/effects; Measurements; Experimental design

\section{Introduction}

Acoustic Doppler velocimeters (ADVs) have been extensively used for both laboratory and field research over the past decades. The ability of ADVs to make nonintrusive, three-dimensional velocity measurements-even in nonclean environments - and their relatively low cost make them a compelling choice in many circumstances. The precision and sources of error of ADVs in the measurements of the mean and higher-order statistics of turbulent flows have been quantified. More precisely, it has been shown that ADVs predict mean and Reynolds shear stresses accurately; however, their precision in measuring normal Reynolds stresses and small-scale turbulence have been questioned (Nikora and Goring 1998; Voulgaris and Trowbridge 1998; Hurther and Lemmin 2008; Khorsandi et al. 2012; Quaresma et al. 2017; Huang et al. 2018; Araujo et al. 2019). The sources of noise are divided into different categories, including (i) sampling error related to resolving phase shifts, which is not flow related; (ii) Doppler noise, which is intrinsic to the technique and is mainly caused by turbulence and particle scattering in the sampling volume; and (iii) error due to the mean velocity shear in the sampling volume (Lohrmann et al. 1994; Lhermitte and Lemmin 1994; Voulgaris and Trowbridge 1998; Hurther and Lemmin 2008; Li et al. 2008).

Doppler noise is the most significant source of noise in turbulence measurements (Garbini et al. 1982; Lohrmann et al. 1994). It adds a positive bias to the root-mean-square (RMS) velocities, resulting in the overestimation of such

Corresponding author: Babak Khorsandi, b.khorsandi@aut.ac.ir measurements (Voulgaris and Trowbridge 1998). Doppler noise is characterized as (i) a white noise, meaning that it has a flat power spectral density; (ii) unbiased, and as a result not influencing the mean velocity; (iii) statistically independent of velocity fluctuations; and (iv) uncorrelated from one receiver to the next (Lohrmann et al. 1994; Hurther and Lemmin 2008).

Based on characteristics (iii) and (iv), the measured variance can be written as the superposition of the true velocity variance and noise variance. Given this, Voulgaris and Trowbridge (1998) showed that the noise contribution to each velocity component depends on the transformation matrix, which transforms the signal measured along the ADV's bistatic axes to orthogonal axes. For a Sontek ADV, they calculated that the noise contribution is $O\left(10^{-2}\right)$ to the covariance, and $O\left(10^{1}\right)$, $O\left(10^{1}\right)$, and $O\left(10^{-1}\right)$ to the variances measured in the $x, y$, and $z$ directions of the probe, respectively.

The ADV probe consists of a transmitter that transmits acoustic pulses and three or four receivers, which record the echoes reflected off suspended particles in the flow. The ADV output is the velocity based on the Doppler phase shift of the acoustics pulses. The sampling volume of the ADV is the volume in which the velocity of scatterers in the fluid is measured and is located roughly $50 \mathrm{~mm}$ below the transmitter (Nortek 2018). The sampling volume is approximately cylindrical and formed by the intersection of the transmit and receive beams. The user-adjustable height of the sampling volume (3-9 mm for a Nortek ADVLab) is determined by the length of the transmit pulse and the width of the receive window. Its width is fixed and is approximated by the diameter of the transmit pulse/ceramic transmitter $(\sim 6 \mathrm{~mm}$ ) (Lohrmann et al. 1994; Voulgaris and Trowbridge 1998). That said, the sampling volume is not a perfectly symmetrical cylinder, as its 
horizontal extent is slightly different for each of the receivers; however, these differences were shown to be negligible by Lohrmann et al. (1994).

ADVs have larger sampling volumes than laser Doppler velocimeters as sound waves have much larger wavelengths than light waves (Dombroski and Crimaldi 2007). The relatively large sampling volume of the ADV may result in certain complications in measurements of mean and turbulence statistics, including (i) spatial averaging of the particle velocities in the sampling volume, causing attenuation of the signal (Garbini et al. 1982 and Hurther and Lemmin 2008) and affecting both the mean velocity and velocity variance estimates; (ii) the presence of mean velocity shear within the sampling volume, which may result in spectral broadening and an increase in the velocity variance (Lhermitte and Lemmin 1994; Voulgaris and Trowbridge 1998; McLelland and Nicholas 2000; Hurther and Lemmin 2008; Li et al. 2008); and (iii) underresolution of turbulent velocity fluctuations of length scales that are smaller than the sampling volume's transverse length (Hurther and Lemmin 2008; Aberle et al. 2017). The contribution of mean velocity shear in the sampling volume to the velocity variance can be estimated by $\sigma_{\mathrm{ms}}^{2}=(\Delta U)^{2} / 3$, where $\Delta U$ is the variation of mean velocity across the sampling volume (Lhermitte and Lemmin 1994; Voulgaris and Trowbridge 1998; McLelland and Nicholas 2000), and the subscript "ms" denotes "mean shear."

The size of the sampling volume may limit an ADV's accuracy in measuring the turbulence microscales, especially at high Reynolds numbers. However, as long as the integral length scale of the flow is much larger than the characteristic length of the sampling volume (as particle velocities within the sampling volume have high correlations), it is expected that spatial averaging will not significantly affect large-scale turbulence quantities such as RMS velocities. (The characteristic length herein is defined as the transverse length or the diameter of the volume cylinder $\sim 6 \mathrm{~mm}$.) Therefore, the user adjustable sampling volume height can be reduced (down to the minimum of $3 \mathrm{~mm}$ ) to accurately capture the turbulence statistics or make near-boundary measurements, if necessary.

Besides the importance of the characteristic length of the sampling volume relative to the length scale of the flow, the size of sampling volume may also have an effect on the noise. Spatial averaging can result in a reduction in Doppler noise (Lohrmann et al. 1994) whereas the mean velocity shear (when present) adds a bias to the velocity variance (Lhermitte and Lemmin 1994). Therefore, reducing the sampling volume height (to capture the integral or smaller scales of the flow, or to measure close to boundaries, for example) can increase the Doppler noise and decrease the error due to mean velocity shear. However, the overall effect of sampling volume size on the noise remains unknown. The increased noise due to the smaller sampling volumes can particularly influence the turbulence measurements conducted by the relatively new Nortek Vectrino profiler, which measures the velocity in cells as small as $1-4 \mathrm{~mm}$ in height.

Despite acknowledgments of the measurement error related to the size of the sampling volume of the ADV in several studies (e.g., Garbini et al. 1982; Lhermitte and Lemmin 1994;
Hurther and Lemmin 2008; Voulgaris and Trowbridge 1998; McLelland and Nicholas 2000) and its effect on the Doppler noise (Lohrmann et al. 1994), no thorough studies have been conducted on the effect of the sampling volume size on the mean and turbulence statistics measured by ADVs. Moreover, ADVs have been almost exclusively benchmarked in openchannel flows (Lhermitte and Lemmin 1994; Voulgaris and Trowbridge 1998; McLelland and Nicholas 2000; Hurther and Lemmin 2001; Valero and Bung 2018). Benchmarking ADVs in free-shear flows, like turbulent jets, which have been extensively studied using other measurement techniques such as hot-wire/film anemometry and laser Doppler anemometry, can further validate the accuracy of ADVs.

The objective of the present study is to determine the effect of sampling volume size on the mean and turbulence statistics of axisymmetric turbulent jets at Reynolds numbers of 10000 and 13000 . The measurements are conducted using three sampling volume heights of 3.1, 6.1, and $9.1 \mathrm{~mm}$.

\section{Experimental setup}

The schematic of the experimental setup is shown in Fig. 1. The experiments were conducted in a $1 \mathrm{~m} \times 1.7 \mathrm{~m} \times 0.54 \mathrm{~m}$ basin that is connected to a $6 \mathrm{~m} \times 0.54 \mathrm{~m} \times 0.54 \mathrm{~m}$ flume filled with water. The water depth was maintained constant at $0.5 \mathrm{~m}$ during experiments. A jet of circular cross section (made of brass tubing) having a diameter $(D)$ of $0.01 \mathrm{~m}$ was installed at the middepth of the basin. The jet fluid (water) was supplied from a constant-head (polyethylene) tank, having a volume of $0.1 \mathrm{~m}^{3}$ with a $2.8-\mathrm{m}$ elevation. The water level in the tank was kept constant by continuously supplying water to it from the end of flume. The jet flowrate was controlled by a ball valve and measured using a (Georg Fischer d32 DN 25) rotameter. The flowrate was set to generate jets at two Reynolds numbers $\left(\operatorname{Re} \equiv U_{j} D / \nu\right.$, where $U_{j}$ is the exit velocity of jet nozzle and $\nu$ is the kinematic viscosity of water) of 10000 and 13000 . The aforementioned Reynolds numbers were chosen as the former is the minimum one above the mixing transition (Dimotakis 2000), and the latter one corresponds to the maximum flow rate achievable in our apparatus. Different jet Reynolds numbers allow the comparison between measurements conducted at various turbulence levels. The measurements were made using a Nortek 10-MHz ADVLab acoustic Doppler velocimeter. The $x, y$, and $z$ directions of the ADV probe were aligned with the axial ( $u$ velocity), horizontal radial ( $v$ velocity), and vertical radial ( $w$ velocity) directions of the jet, respectively. During the measurements, the sampling frequency was set to its maximum $(25 \mathrm{~Hz})$. Talcum powder was used as a scatterer to increase the signal-to-noise ratio (SNR) of the measurements (Moeini et al. 2020a). Three sampling volume heights of $3.1,6.1$, and $9.1 \mathrm{~mm}$ (maximum) were tested to assess the effect of sampling volume size on the measurements. The transmit pulse length was set to $2.4 \mathrm{~mm}$ (maximum) for all three sampling volume heights. The velocity range of the ADV was set to $\pm 10, \pm 30$, or $\pm 100 \mathrm{~cm} \mathrm{~s}^{-1}$, to span the full range of the measured velocities. The centerline of the jet was determined by fitting a Gaussian distribution to its measured horizontal and vertical profiles, and the center was interpolated 
a)
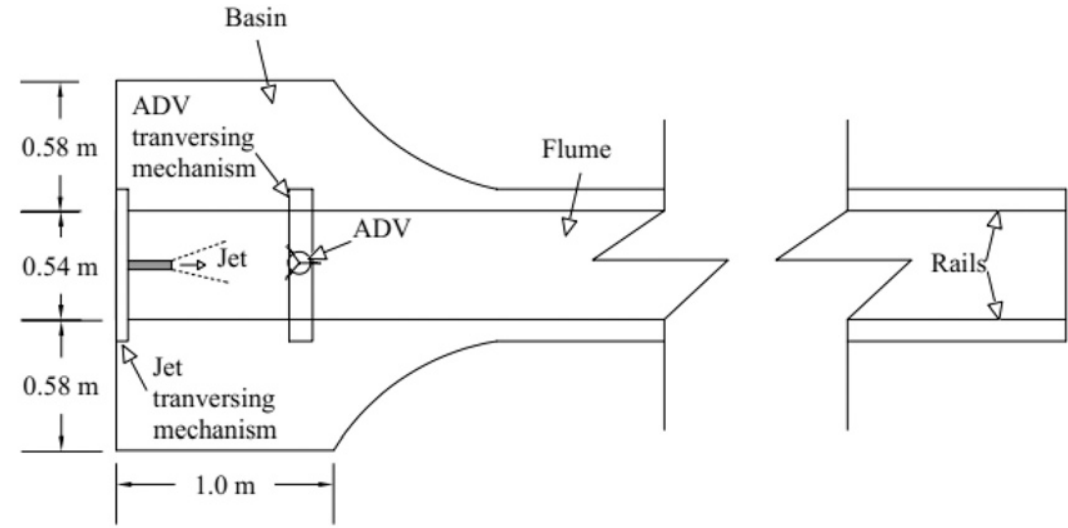

b)
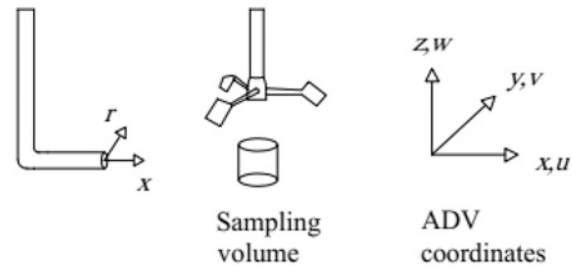

FIG. 1. Schematic diagrams of (a) the top view of the flume, measurement apparatus, and jet facility, and (b) the jet, sampling volume, and ADV probe (not to scale).

from the curve fit. 60000 data points were recorded for each experiment, corresponding to a total recording time of $40 \mathrm{~min}$, which ensured convergence of statistics up to fourth-order moments.

\section{Results}

The mean and turbulence statistics of the jet at Reynolds numbers of 10000 and 13000 measured by the ADV at different sampling volume heights of $3.1,6.1$, and $9.1 \mathrm{~mm}$ will be presented in this section. The results will be compared to those of the other studies including those employing acoustic Doppler velocimetry (Khorsandi et al. 2013), stationary hotwire anemometry (SHWA; Hussein et al. 1994), flying hot-wire anemometry (FHWA; Panchapakesan and Lumley 1993), and laser Doppler anemometry (LDA; Hussein et al. 1994; Darisse et al. 2015). The work of Panchapakesan and Lumley (1993), Hussein et al. (1994), and Darisse et al. (2015) will be abbreviated as PL, HCG, and DLB, respectively, in the figures.

Before presenting the results, it is important to first examine how the spatial and temporal averaging of the ADV affects the statistics. If the ratios of sampling volume (transverse) length to the longitudinal integral length scale (SV/ILS) and sampling interval to the integral time scale (SI/ITS) are much less than one, particle velocities within the sampling volume have high correlations. Therefore, it is expected that the effects of spatial and temporal averaging will be minimal and not result in significant damping of the true signal. As a result, the large-scale turbulence quantities, such as the (true) velocity variances are not significantly affected. However, this averaging may also result in noise reduction (assuming the noise is uncorrelated from one sample to the next).
The SV/ILS and SI/ITS ratios at the centerline of the jet at $\operatorname{Re}=10000$ are plotted as a function of $x / D$ in Fig. 2. Here, SV $\sim 6 \mathrm{~mm}$ and SI $=0.04 \mathrm{~s}$, which correspond to the transverse length of the sampling volume and the inverse of the sampling frequency of $25 \mathrm{~Hz}$, respectively. The ITS is calculated from the integral of the autocorrelation function. The ILS is obtained using Taylor's hypothesis [ILS $=U_{\mathrm{CL}}(x) \times$ ITS, where $U_{\mathrm{CL}}(x)$ is the centerline mean velocity; Tennekes and Lumley 1972; Moeini et al. 2020b]. It can be seen that these ratios are smaller than 1 over the entire range of measurements and decrease with increasing downstream distance. Note that SV/ILS and SI/ITS follow $x^{-0.9}$ and $x^{-1.9}$ power-law decays, which are consistent with theoretical predictions of $x^{-1}$ and $x^{-2}$, respectively (Pope 2000). Therefore, it is expected that the effect of damping on the true signal will be negligible, especially when the ratios decrease at larger downstream distances.

The downstream variation of the (inverse of the) centerline mean axial velocity is presented in Fig. 3. The inverse of the axial mean velocity at the centerline varies linearly with downstream distance in the self-similar region: $U_{J} / U_{\mathrm{CL}}=[(x-$ $\left.\left.x_{0}\right) / D\right] / B$, where $x_{0}$ is the virtual origin and $B$ is the decay rate, which is the inverse of the slope (Pope 2000). It can be seen that the centerline axial mean velocities do not exhibit any significant dependence on the sampling volume size. This may be due to the lack of mean velocity shear at the jet centerline. The velocities at $x / D>90$, especially those for $\mathrm{Re}=10000$, deviate from the straight line due to the return flow resulting from the finite size of the enclosure, as discussed by Hussein et al. (1994). Flow visualization experiments confirmed the existence of return flow in the basin and flume.

Table 1 compares $B, x_{0}$, and the spreading rates $S$ against those of other studies. The spreading rate is defined as $S=d r_{1 / 2} / d x$, 
a)

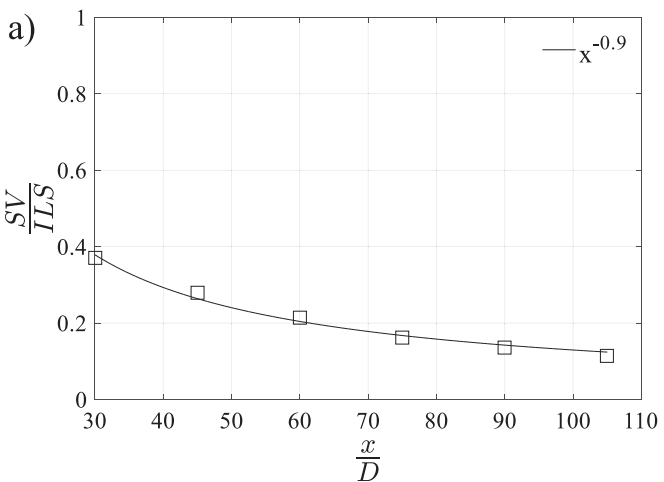

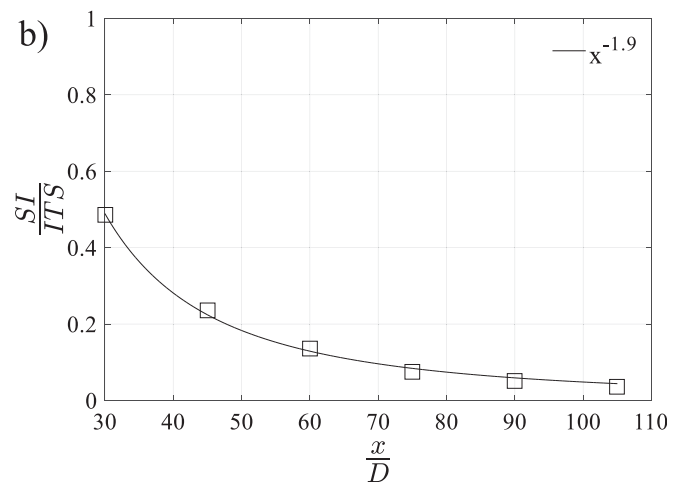

FIG. 2. Downstream variation of (a) the ratio of sampling volume size $(\sim 6 \mathrm{~mm})$ to the integral length scale of the jet $(\mathrm{Re}=10000), \mathrm{SV} / \mathrm{ILS}$, and $(\mathrm{b})$ the ratio of sampling interval $(=0.04 \mathrm{~s})$ to the integral time scale of the same flow, SI/ITS. The solid lines represent the best-fit power laws to the data.

where $r_{1 / 2}$ is the jet's half-width, which is defined to be the radial position at which the mean velocity falls to half of its centerline value. $B$ and $x_{0}$ were calculated from the method of least squares using the aforementioned equation fitted to data measured using different sampling volume sizes. The data over the range $20<x / D<90$ were used to calculate the values of $B$ and $x_{0}$, and the value of $S$ is obtained from the mean velocity profiles at $x / D=30,60$, and 90 . There is reasonable agreement between the data of the current study and those of the other studies given the difference in Reynolds numbers and other parameters in these experiments. The slightly smaller decay rate is due to the return flow, which results in a faster decay of the downstream velocities. Also note that $x_{0}$ depends on the initial conditions (Pope 2000). (Note that $S$ is not reported at $\mathrm{Re}=13000$ case because the radial velocity profiles were not measured for this case.)

The radial profiles of axial mean velocity of the jet at $\mathrm{Re}=$ 10000 were measured at $x / D=30,60$, and 90 using different sampling volume heights and are plotted in Fig. 4. Figure 4a shows that the jet decays and spreads (such that its mean velocity shear decreases) with increasing the downstream distance. The profiles are replotted using self-similar normalization in Figs. 4b-d. The shapes of the profiles agree well with those of the other studies. Despite the major variations of the mean velocity shear from $x / D=30-90$ (as shown in Fig. 4a) as well as the velocity gradient variations across the profiles, no significant difference is observed between the mean velocities measured at the three sampling volume sizes. This suggests that the mean statistics are effectively independent of the instrument's sampling volume size (at least in the flow studied herein). As remarked before, the underestimated velocities at the edge of the jet at $x / D=90$ are due to the return flow (Hussein et al. 1994), which is common in enclosures and for which the effect is more significant when velocities are smallest, which is the case at the farthest downstream distance.

The velocity variance along the jet centerline will be presented next. Note that due to the zero velocity gradient (in the radial direction) along the centerline, the mean velocity shear error should have a negligible effect on the velocity variance at that location. Given that SV/ILS is also small over the measurement range, the true velocity variance will not be significantly damped due to spatial averaging effects. Therefore, it is expected that any difference in variance measured at different sampling volumes in the following figures is solely due to Doppler noise.

Figure 5 plots the velocity variances measured using different sampling volume sizes along the centerline of jet for $\mathrm{Re}=$ 10000 and 13000 . This quantity is normalized by the square of the nozzle exit velocity in Figs. 5a,c,e and mean centerline velocity in Figs. 5b,d,f. The velocity variance normalized by the square of mean velocity asymptotes to a constant in the selfsimilar region given that both the RMS and mean velocities decay as $x^{-1}$. Overall, one observes that the ADV overestimates the $u$ and $v$ velocity variances (measured along the $x$ and $y$ directions of the probe, respectively) compared to the results employing other measurement techniques. However, the $w$ velocity variance, which is measured along the $z$ direction of the probe, exhibits results that are consistent with those of prior researchers. It should be noted that the $v$ and $w$ velocity

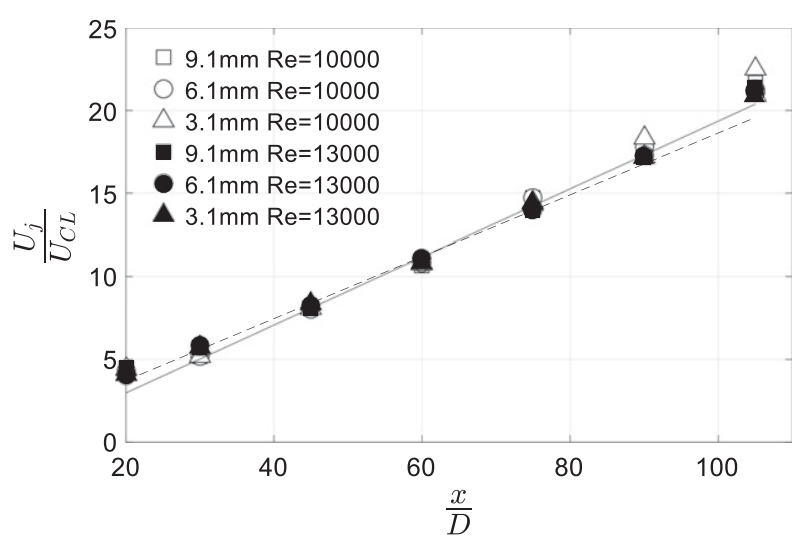

FIG. 3. The downstream variation of the inverse of the centerline mean axial velocity at different sampling volume sizes and Reynolds numbers. The solid and broken lines are linear least squares fits to the $\mathrm{Re}=10000$ and 13000 data, respectively. 
TABLE 1. Comparison of the mean velocity parameters for the turbulent axisymmetric jet of the present study and those of other studies.

\begin{tabular}{lcccccc}
\hline \hline & $\begin{array}{c}\text { Present } \\
\text { work ADV }\end{array}$ & $\begin{array}{c}\text { Present } \\
\text { work ADV }\end{array}$ & $\begin{array}{c}\text { Khorsandi et al. } \\
(2013) \text { ADV }\end{array}$ & $\begin{array}{c}\text { Panchapakesan and Lumley } \\
\text { (1993) FHWA }\end{array}$ & $\begin{array}{c}\text { Hussein et al. } \\
\text { (1994) SHWA }\end{array}$ & $\begin{array}{c}\text { Hussein et al. } \\
\text { (1994) LDA }\end{array}$ \\
\hline $\operatorname{Re}$ & 10000 & 13000 & 10600 & 11000 & 95500 & 95500 \\
$x_{0} / D$ & 5.5 & 0.15 & 5.5 & 0 & 2.7 & 4 \\
$B$ & 5.0 & 5.4 & 5.43 & 6.06 & 5.9 & 5.8 \\
$S$ & 0.101 & - & 0.099 & 0.096 & 0.102 & 0.094 \\
\hline
\end{tabular}

variances should be the same due to the symmetry of the axisymmetric jet. The overestimation of the $u$ and $v$ velocity variances, as well as the difference between the $v$ and $w$ velocity variances, is due to the geometrical configuration of the probe, which results in higher levels of noise in the $x$ and $y$ directions relative to the $z$ direction (Voulgaris and Trowbridge 1998; Khorsandi et al. 2012; Moeini et al. 2020a). It is worth remarking that the noise increases as the velocity range setting increases (Voulgaris and Trowbridge 1998). This was further confirmed by additional experiments showing that increasing the velocity range slightly increases the velocity variance due to the increased noise level. For the centerline velocity measurements, the velocity range was set to $\pm 100 \mathrm{~cm} \mathrm{~s}^{-1}$ for $x / D<60$, and to $\pm 30 \mathrm{~cm} \mathrm{~s}^{-1}$ for $x / D \geq 60$. The slight decrease in the turbulence intensities from $x / D=45$ to 60 in (as seen in Figs. 5b,d) may be due to the change in the velocity range.
Regarding the effect of sampling volume size on the measurements, it can be seen that the smaller sampling volume heights (especially, $3.1 \mathrm{~mm}$ ) result in larger velocity variances. This is attributed to the fact that spatial averaging over larger sampling volumes results in a reduction of the Doppler noise while it has no significant effect on the true velocity variance because SV/ILS is small. Moreover, the difference between the velocity variances measured using different sampling volume heights reduces with increasing downstream distance because both the turbulence and the Doppler noise (which is caused by the turbulence) decay downstream. In addition, the velocity variances for the $\mathrm{Re}=13000$ case are initially higher than those for $\mathrm{Re}=10000$. However, farther downstream, the data become indistinguishable as the turbulence and Doppler noise decay. The effect of sampling volume size is more significant for the $u$ and $v$ velocity variances (which are contaminated by
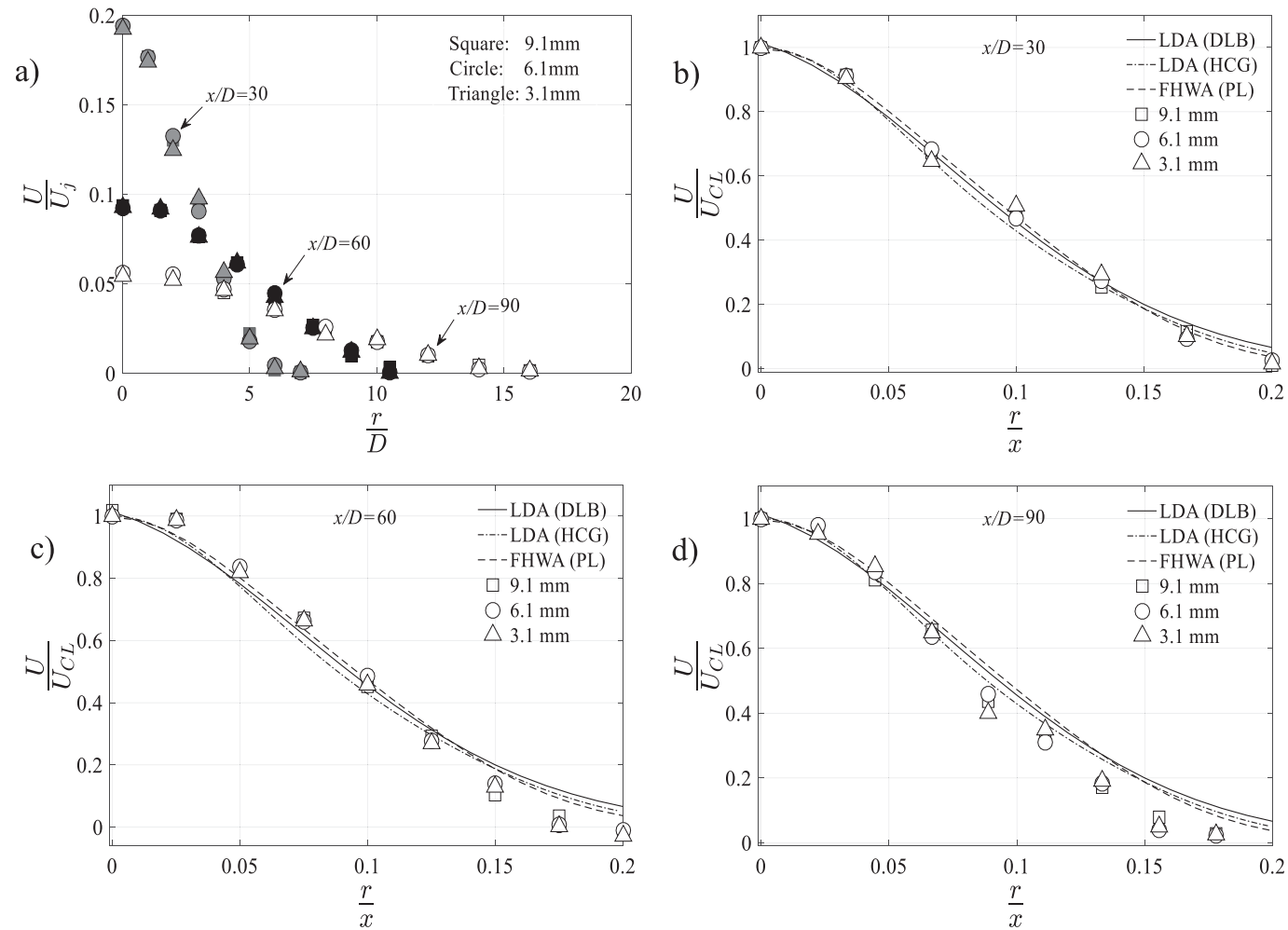

FIG. 4. Radial profiles of normalized axial mean velocity of the jet at $\mathrm{Re}=10000$ measured using different sampling volumes at (a) $x / D=30,60$, and 90 (non-self-similar normalization), (b) $x / D=30$, (c) $x / D=60$, and (d) $x / D=90$, and compared with other studies. 

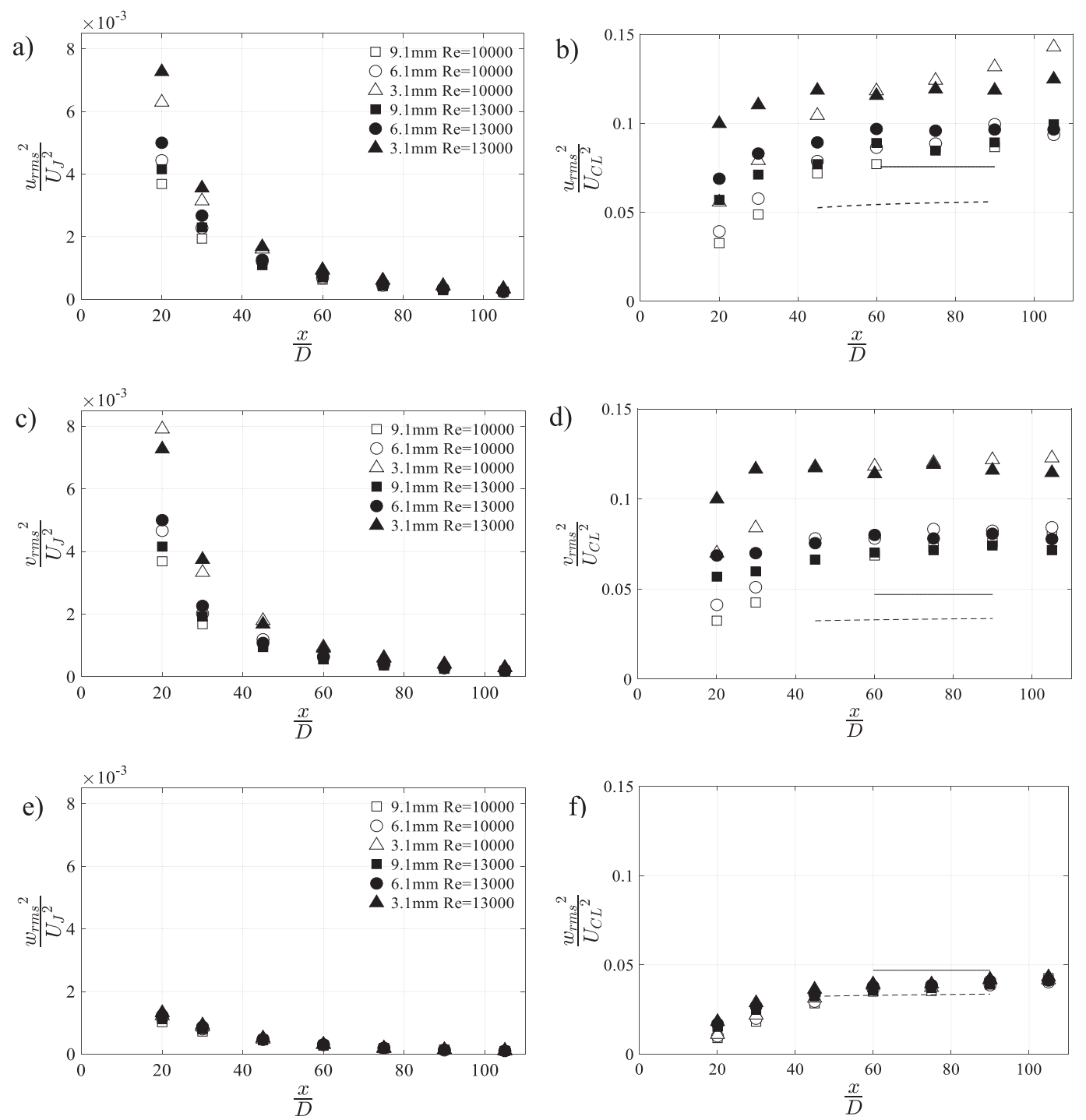

FIG. 5. Downstream evolution of the centerline velocity variance at $\mathrm{Re}=10000$ and 13000 measured using different sampling volume sizes. (a) $u$, (c) $v$, and (e) $w$ velocity variances, normalized by the square of nozzle exist velocity; (b) $u$, (d) $v$, and (f) $w$ velocity variances, normalized by the square of mean centerline velocity. The solid and broken lines correspond to curve fits to the data of Hussein et al. (1994) and Panchapakesan and Lumley (1993), respectively.

higher Doppler noise) compared to the $w$ velocity variance. As can be observed in Figs. 5e and 5f, the $w$ velocity variance has low noise, so that the spatial averaging does not significantly affect its value.

Figure 6 depicts the radial profiles of the normalized velocity variances of the jet $(\mathrm{Re}=10000)$ measured at $x / D=60$ using different sampling volume heights. The general shape of the profiles is comparable with prior results employing other measurement techniques. But the $u$ and $v$ velocity variances are, once again, overestimated, especially using the smallest sampling volume height of $3.1 \mathrm{~mm}$. On the other hand, the $w$ velocity variance agrees well with those of other studies, and especially with that of Panchapakesan and Lumley (1993).
Overall, it can be seen that the larger sampling volumes result in smaller velocity variances, especially for the $u$ and $v$ velocities. This is due to the fact that when the sampling volume size increases, the number of scattered particles in the sampling volume rises and, therefore, the output velocity is averaged over more particles (of high correlations), resulting in reduced Doppler noise. Note that the damping of the true variance is negligible because the sampling volume size is almost one order of magnitude smaller than the integral length scale of the flow. As the turbulence is reduced toward the edge of the jet, the Doppler noise reduces and the velocity variances measured using different sampling volumes merge. The $w$ velocity variance has less noise and its profile is thus not 

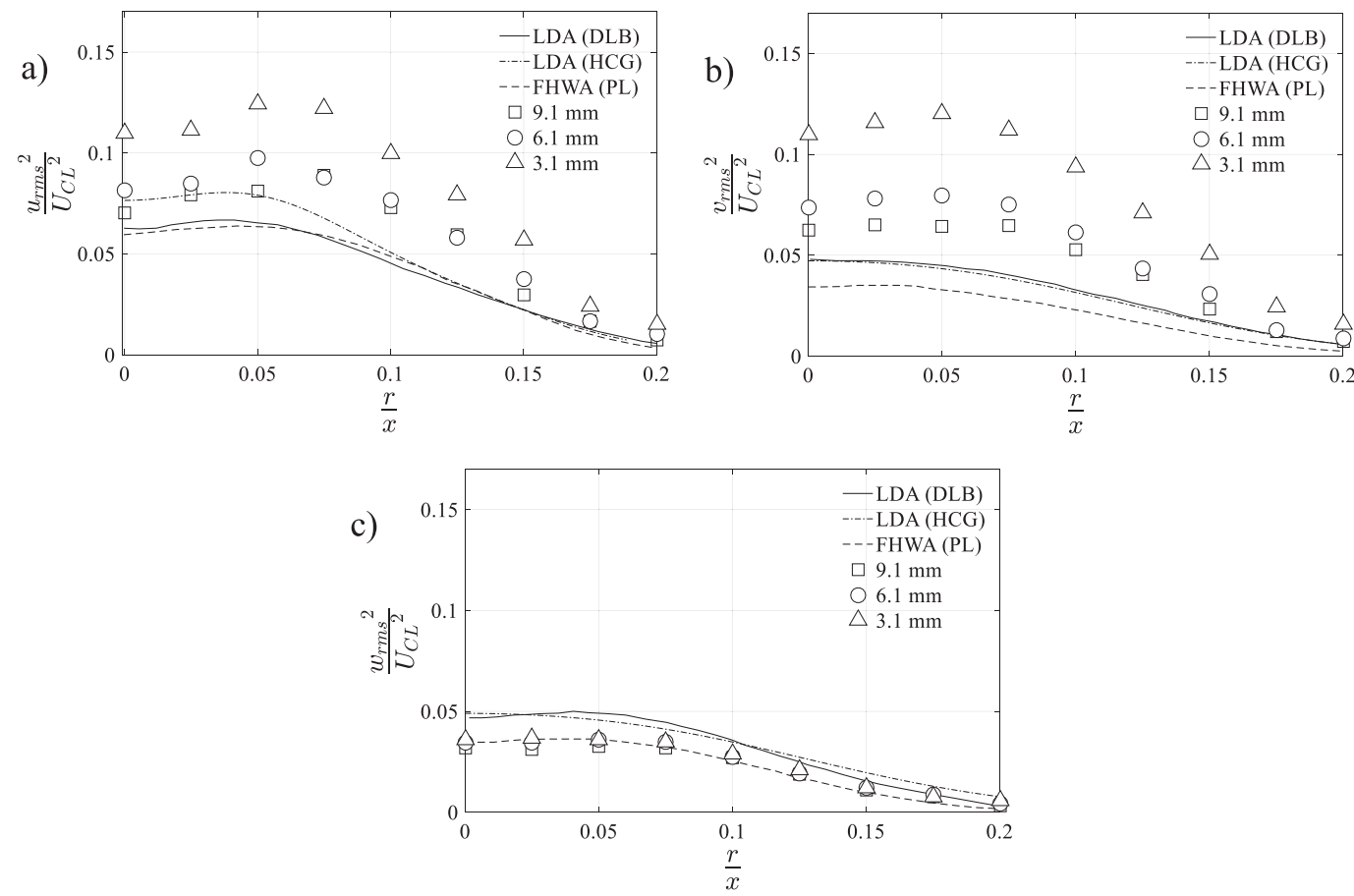

FIG. 6. Radial profiles of the normalized velocity variances $(\mathrm{Re}=10000)$ measured at $x / D=60$ using different sampling volume heights. (a) $u$, (b) $v$, and (c) $w$ velocity variances, normalized by the square of the mean centerline velocity.

significantly affected by the size of the sampling volume. The profiles measured at $x / D=30$ and 90 (not shown) show similar trends when the height of the sampling volume was varied from 3.1 to $9.1 \mathrm{~mm}$. It should be remarked that the velocity range (for profiles measured at $x / D=60$ ) was set to $\pm 30 \mathrm{~cm} \mathrm{~s}^{-1}$ over the range $0 \leq r / x \leq 0.15$, and to $\pm 10 \mathrm{~cm} \mathrm{~s}^{-1}$ for $r / x>0.15$. Hence, the same velocity range setting was used for most of the measurements.

Figure 7 plots velocity spectra measured at the centerline of the jet $(\operatorname{Re}=10000)$ at $x / D=90$ for three different sampling volumes. One observes that the spectra measured using smaller sampling volumes have higher noise levels. On the other hand, as the size of the sampling volume increases, the inertial subrange slope decreases and more closely approaches a value of $-5 / 3$. The reason, as explained previously, is that the measurements using smaller sampling volumes are more affected by the noise. The $w$ spectra, as expected from their lower noise levels, are less influenced by the size of the sampling volume.

\section{Discussion}

Lhermitte and Lemmin (1994), Voulgaris and Trowbridge (1998), Hurther and Lemmin (2008), and McLelland and Nicholas (2000) theoretically showed that velocity shear in the sampling volume broadens the spectra and results in noise variances that are proportional to the square of the difference in the mean velocity across the transmitted pulse. However, the present observations suggest that error due to mean velocity
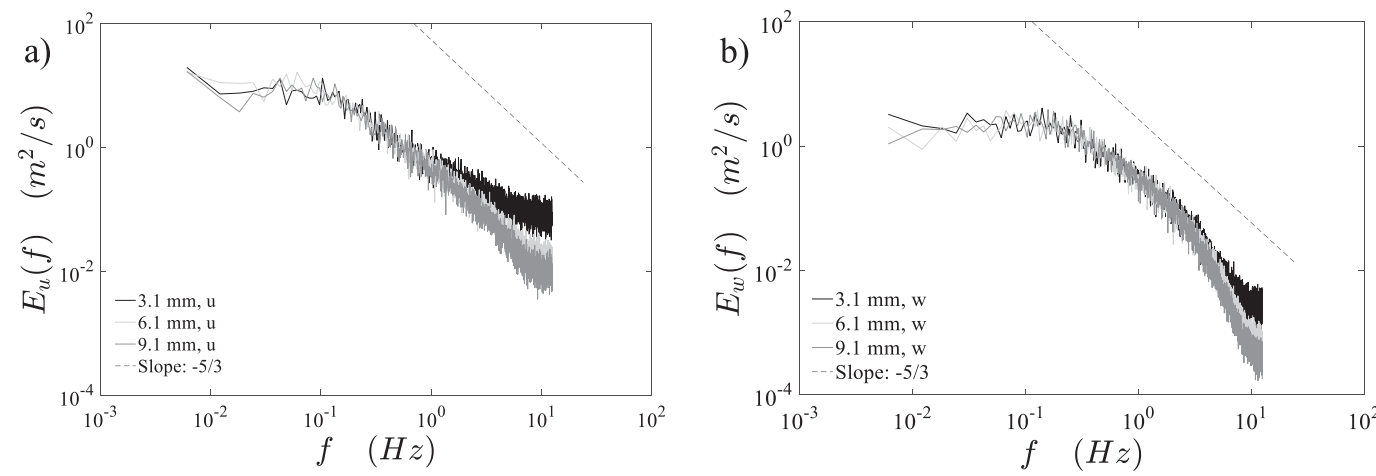

FIG. 7. (a) Axial ( $u$ ) and (b) vertical ( $w$ ) velocity spectra measured at the jet centerline and $x / D=90$ for the three different sampling volumes considered. $\mathrm{Re}=10000$. The velocity range was set to $\pm 30 \mathrm{~cm} \mathrm{~s}^{-1}$. The dashed line denotes a $-5 / 3$ slope. 

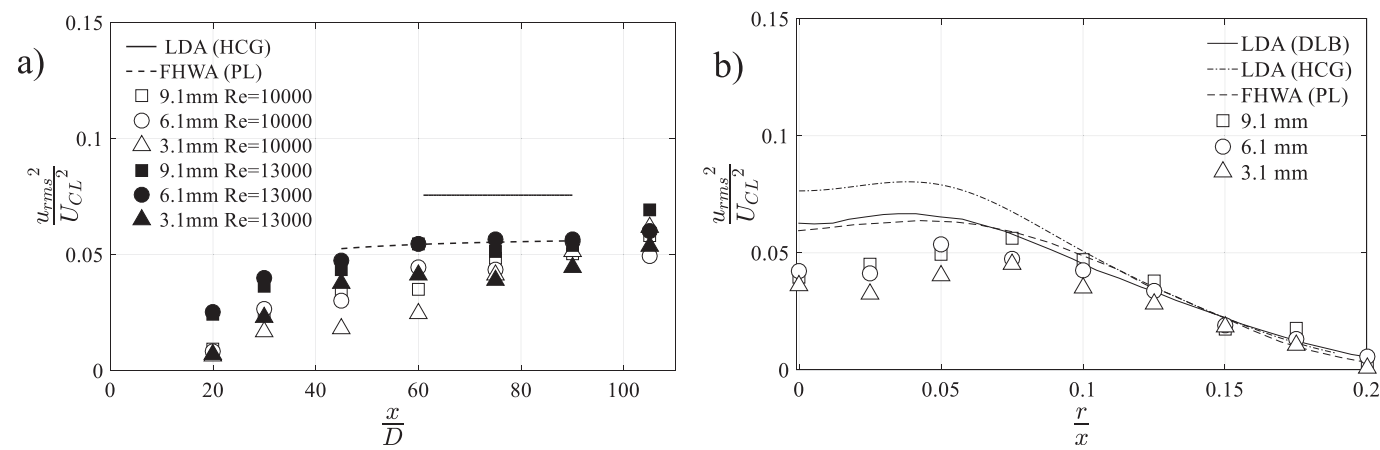

FIG. 8. (a) Downstream evolution of the centerline velocity variance of jet at $\mathrm{Re}=10000$ and 13000 measured using different sampling volume sizes after application of the noise-reduction method of Khorsandi et al. (2012). (b) Radial profiles of the normalized axial velocity variances of the jet $(\operatorname{Re}=10000)$ measured at $x / D=60$ using different sampling volume sizes after application of the noise-reduction method of Khorsandi et al. (2012).

shear does not seem to be significant since (i) increasing the sampling volume size (which results in higher shear across the volume) generally resulted in a decrease in the velocity variances; (ii) with increasing downstream distance, the mean velocity profiles decrease in slope (see Fig. 4a), that is, the mean velocity shear in the sampling volume decreases, however, this does not result in a reduction in noise; and (iii) the difference between velocity variances measured using different sampling volumes does not seem to be affected by the mean velocity shear or the change of slope across the profile; for example, the velocity variances do not change significantly for $0 \leq r / x \leq 0.1$, where the mean velocity shear varies from zero to its largest values. Therefore, the effect of error due to the mean velocity shear appears to be negligible relative to the Doppler noise, at least in the flows studied herein.

To further examine the effect of the noise on the velocity variances measured using different sampling volume sizes, postprocessing methods were applied to the data. The phasespace thresholding method (Goring and Nikora 2002; modified by Wahl 2003), which is implemented in the WinADV software, was used to despike the data. However, the filter did not considerably improve the RMS velocities measured at different sampling volumes, as the signal quality in the present experiments was high. For example, after despiking, the RMS velocities measured at the centerline of the jet $(\mathrm{Re}=10000)$ at $x / D=60$, decreased by less than $1.5 \%$ for different sampling volume heights. Note that spikes usually occur in poor measurement environments [e.g., when the velocities exceed the preset velocity range or when there are backscattered signals from boundaries (Goring and Nikora 2002)], and therefore, are not inherent to the technique (Doroudian et al. 2010). In addition to despiking, denoising techniques (e.g., Hurther and Lemmin 2001; Khorsandi et al. 2012; Thomas et al. 2017; Moeini et al. 2020a) can be applied for the correction of the statistics measured using ADVs.

Khorsandi et al. (2012) presented a noise-reduction method for axisymmetric flows. Their method is based on the assumptions that variances of the $v$ and $w$ velocities must be similar due to the axisymmetry of the flow, and that the variance of $w$ has negligible noise. The noise in the $u$ velocity variance can be estimated by subtracting the variance of the $w$ velocity from the variance of the $v$ velocity and then converting the result to the variance of noise in the $x$ direction using the ADV's transformation matrix. The noise can subsequently be subtracted from the measured variance of the $u$ velocity. This method has been applied to our data measured using various sampling volumes and the results are presented in Fig. 8. One observes that the agreement of the measurements of the variance of the $u$ velocity recorded using different sampling volumes are improved (especially at $x / D>60$ ) once the method has been applied. This confirms that the difference between the variance measured at various sampling volumes is mainly due to the Doppler noise. [Note that some of the observed differences between the variance at $\mathrm{Re}=10000$ and $\mathrm{Re}=13000$ in Fig. $8 \mathrm{a}$ may be attributed to the variations in the mean velocities, as seen in Fig. 3. It should also be emphasized that the underestimation of the data in the vicinity of the jet centerline in Fig. $8 \mathrm{~b}$ is probably because the normalized variances have not yet reached their asymptotic values, which are only achieved beyond $x / D=70-100$ depending on the initial conditions and measurement technique (Panchapakesan and Lumley 1993; Hussein et al. 1994; Khorsandi et al. 2013).] The results and discussions presented here reinforce the necessity of applying noise-reduction methods [such as those of Hurther and Lemmin (2001), Khorsandi et al. (2012), or Moeini et al. (2020a)] to the data, especially if measurements are conducted using small sampling volumes.

\section{Conclusions}

To study the effect of sampling volume size of an ADV on measurements of turbulence statistics, experiments with different ADV sampling volume heights were conducted in an axisymmetric turbulent jet. The results showed that the mean velocities were not notably affected by the size of the sampling volume. However, reducing the sampling volume size resulted in an increase in (Doppler noise and therefore) the variances of the $u$ and $v$ velocities, while its effect on the $w$ velocity variance was negligible. Therefore, the principal conclusion of this work is that as long as the characteristic length of sampling volume is significantly smaller than the integral length scale of flow, 
increasing the sampling volume size (which is typically associated with increasing the volume over which highly correlated scatterers are spatially averaged) can reduce Doppler noise, resulting in more accurate measurements. Moreover, applying the noise-reduction method of Khorsandi et al. (2012) to the data resulted in improved agreement of the variance of the $u$ velocity measured using different sampling volume sizes, from which one may conclude that the difference between variances measured at different sampling volume sizes is mainly due to the Doppler noise. Applying noise-reduction methods to the data is especially important when the data is acquired using small sampling volumes. Furthermore, noise due to the mean velocity shear, even in regions of the largest velocity gradient along the jet radial profile, is found to be negligible in the present experiments. For future work, it is recommended to investigate the effect of cell sizes of the relatively new Nortek Vectrino Profiler on turbulence measurements. Based on the results presented here and given the small cell size of the Vectrino Profiler, one might expect that its turbulence measurements might suffer from higher levels of noise than that observed in the present study.

Acknowledgments. The authors thank the Natural Sciences and Engineering Research Council (NSERC) of Canada for funding part of this work: Grant RGPIN-2018-05848 (LM).

Data availability statement. The dataset on which this paper is based is too large to be retained or publicly archived with available resources. Documentation and methods used to support this study are available from the corresponding author upon reasonable request.

\section{REFERENCES}

Aberle, J., C. D. Rennie, D. M. Admiraal, and M. Muste, 2017: Instrumentation and Measurement Techniques. Vol. II, Experimental Hydraulics, Methods, Instrumentation, Data Processing and Management, CRC Press, 906 pp.

Araujo, M. A. V. C., B. J. Araujo, and B. Greenwood, 2019: Acoustic Doppler velocimetry measurements of flow over a backward-facing step. J. Hydraul. Res., 58, 850-858, https:// doi.org/10.1080/00221686.2019.1671521.

Darisse, A., J. Lemay, and A. Benaïssa, 2015: Budgets of turbulent kinetic energy, Reynolds stresses, variance of temperature fluctuations and turbulent heat fluxes in a round jet. J. Fluid Mech., 774, 95-142, https://doi.org/10.1017/jfm.2015.245.

Dimotakis, P. E., 2000: The mixing transition in turbulent flows. J. Fluid Mech., 409, 69-98, https://doi.org/10.1017/ S0022112099007946.

Dombroski, D. E., and J. P. Crimaldi, 2007: The accuracy of acoustic Doppler velocimetry (ADV) measurements in turbulent boundary layer flows over a smooth bed. Limnol. Oceanogr. Methods, 5, 23-33, https://doi.org/10.4319/1om.2007.5.23.

Doroudian, B., F. Bagherimiyab, and U. Lemmin, 2010: Improving the accuracy of four-receiver acoustic Doppler velocimeter (ADV) measurements in turbulent boundary layer flows. Limnol. Oceanogr. Methods, 8, 575-591, https://doi.org/10.4319/ lom.2010.8.0575.

Garbini, J. L., F. K. Forster, and J. E. Jorgensen, 1982: Measurement of fluid turbulence based on pulsed ultrasound techniques. Part I. Analysis. J. Fluid Mech., 118, 445-470, https://doi.org/ 10.1017/S0022112082001153.
Goring, D. G., and V. I. Nikora, 2002: Despiking acoustic Doppler velocimeter data. J. Hydraul. Eng., 128, 117126, https://doi.org/10.1061/(ASCE)0733-9429(2002)128: 1(117).

Huang, J. H., H. Ma, J. Guo, D. Dai, and F. Qiao, 2018: Calculation of turbulent dissipation rate with acoustic Doppler velocimeter. Limnol. Oceanogr. Methods, 16, 265-272, https://doi.org/ 10.1002/lom3.10243.

Hurther, D., and U. Lemmin, 2001: A correction method for turbulence measurements with a 3D acoustic Doppler velocity profiler. J. Atmos. Oceanic Technol., 18, 446-458, https://doi.org/10.1175/1520-0426(2001)018<0446:ACMFTM> 2.0.CO;2.

- , and -2008 : Improved turbulence profiling with fieldadapted acoustic Doppler velocimeters using a bifrequency Doppler noise suppression method. J. Atmos. Oceanic Technol., 25, 452-463, https://doi.org/10.1175/2007JTECHO512.1.

Hussein, H. J., S. P. Capp, and W. K. George, 1994: Velocitymeasurements in a high-Reynolds-number, momentumconserving, axisymmetrical, turbulent jet. J. Fluid Mech., 258, 31-75, https://doi.org/10.1017/S002211209400323X.

Khorsandi, B., L. Mydlarski, and S. Gaskin, 2012: Noise in turbulence measurements using acoustic Doppler velocimetry. J. Hydraul. Eng., 138, 829-838, https://doi.org/10.1061/(ASCE) HY.1943-7900.0000589.

__, S. Gaskin, and L. Mydlarski, 2013: Effect of background turbulence on an axisymmetric turbulent jet. J. Fluid Mech., 736, 250-286, https://doi.org/10.1017/jfm.2013.465.

Lhermitte, R., and U. Lemmin, 1994: Open-channel flow and turbulence measurement by high-resolution Doppler sonar. J. Atmos. Oceanic Technol., 11, 1295-1308, https://doi.org/ 10.1175/1520-0426(1994)011<1295:OCFATM>2.0.CO;2.

Li, D. X., M. Muste, and X. K. Wang, 2008: Quantification of the bias error induced by velocity gradients. Meas. Sci. Technol., 19, 015402, https://doi.org/10.1088/0957-0233/ 19/1/015402.

Lohrmann, A., R. Cabrera, and N. C. Kraus, 1994: AcousticDoppler velocimeter (ADV) for laboratory use. Fundamentals and Advancements in Hydraulic Measurements and Experimentation, ASCE, 351-365.

McLelland, S. J., and A. P. Nicholas, 2000: A new method for evaluating errors in a high-frequency ADV measurements. Hydrol. Processes, 14, 351-366, https://doi.org/10.1002/(SICI) 1099-1085(20000215)14:2<351::AID-HYP963>3.0.CO;2-K.

Moeini, M., B. Khorsandi, and L. Mydlarski, 2020a: Effect of acoustic Doppler velocimeter (ADV) sampling frequency on statistical measurements of turbulent axisymmetric jets: An attempt to improve ADV measurements. J. Hydraul. Eng., 146, 04020048, https://doi.org/10.1061/(ASCE)HY.19437900.0001767 .

,-- , and,$- 2020 \mathrm{~b}$ : Effect of coflow turbulence on the dynamics and mixing of a non-buoyant turbulent jet. J. Hydraul. Eng., 147, 04020088, https://doi.org/10.1061/(ASCE)HY.19437900.0001830.

Nikora, V. I., and D. G. Goring, 1998: ADV measurements of turbulence: Can we improve their interpretation? J. Hydraul. Eng., 124, 630-634, https://doi.org/10.1061/(ASCE)07339429(1998)124:6(630).

Nortek, 2018: The comprehensive manual for velocimeters. Nortek, 119 pp., https://www.nortekgroup.com/assets/software/N3015030-Comprehensive-Manual-Velocimeters_1118.pdf.

Panchapakesan, N. R., and J. L. Lumley, 1993: Turbulence measurements in axisymmetric jets of air and helium. Part I. 
Air jet. J. Fluid Mech., 246, 197-223, https://doi.org/10.1017/ S0022112093000096.

Pope, S. B., 2000: Turbulent Flows. Cambridge University Press, $771 \mathrm{pp}$.

Quaresma, A. L., R. M. L. Ferreira, and A. N. Pinheiro, 2017: Comparative analysis of particle image velocimetry and acoustic Doppler velocimetry in relation to a pool-type fishway flow. J. Hydraul. Res., 55, 582-591, https://doi.org/10.1080/ 00221686.2016.1275051.

Tennekes, H., and J. L. Lumley, 1972: A First Course in Turbulence. MIT Press, $300 \mathrm{pp}$.

Thomas, R. E., L. Schindfessel, S. J. McLelland, S. Creëlle, and T. De Mulder, 2017: Bias in mean velocities and noise in variances and covariances measured using a multistatic acoustic profiler: The Nortek Vectrino Profiler. Meas. Sci. Technol., 28, 075302, https://doi.org/10.1088/1361-6501/aa7273.

Valero, D., and D. B. Bung, 2018: Vectrino profiler spatial filtering for shear flows based on the mean velocity gradient equation. J. Hydraul. Eng., 144, 04018037, https://doi.org/10.1061/(ASCE) HY.1943-7900.0001485.

Voulgaris, G., and J. H. Trowbridge, 1998: Evaluation of the acoustic Doppler velocimeter (ADV) for turbulence measurements. J. Atmos. Oceanic Technol., 15, 272-289, https://doi.org/10.1175/ 1520-0426(1998)015<0272:EOTADV>2.0.CO;2.

Wahl, T. L., 2003: Discussion of "Despiking acoustic Doppler velocimeter data" by Derek G. Goring and Vladimir I. Nikora. J. Hydraul. Eng., 129, 484-487, https://doi.org/10.1061/(ASCE) 0733-9429(2003)129:6(484). 